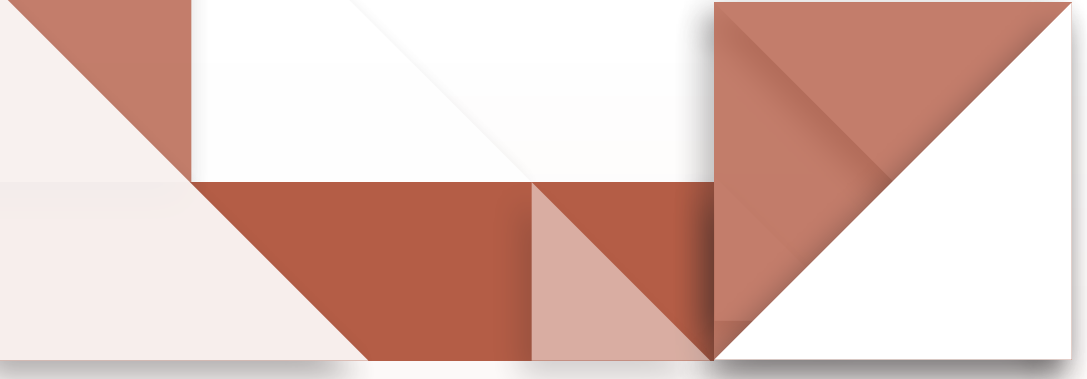

\title{
1) Percepciones y expectativas profesionales en estudiantes de Licenciatura en Física
}

\author{
Mónica Eliana Cardona* \\ Jaime Andrés Carmona* \\ Vanessa Arias Gil***
}

- Professional Perceptions and Expectations of Students in a Physics Bachelor

- Percepções e expectativas profissionais em estudantes de Bacharelado em Física

\section{Resumen}

La identidad profesional docente es un eje fundamental en la formación de profesores. Su estudio desde los primeros niveles de formación permite comprender su configuración e incidencia en las prácticas pedagógicas. El propósito de esta investigación fue caracterizar los rasgos de identidad profesional que tienen los estudiantes de un programa de Licenciatura en Física a partir de sus percepciones y expectativas sobre la profesión docente, así como valorar los aportes del espacio de formación Práctica Pedagógica I en el reconocimiento de la construcción de dicha identidad. El estudio se desarrolló bajo un enfoque biográfico- narrativo y recurrió a la elaboración de cartas y a la autoevaluación como instrumentos para el registro de la información. Como principal hallazgo se informa la transformación de las concepciones de los estudiantes sobre la profesión docente, logrando reconocer los diferentes componentes de la construcción de la identidad y aplicarlos en su reconocimiento como futuros profesores.

Palabras clave:

formación de profesores; práctica pedagógica; prácticum; identidad profesional; profesión docente

Magíster en Educación en Ciencias Naturales. Profesora, Facultad de Educación, Departamento de Enseñanza de las Ciencias y las Artes, Universidad de Antioquia, Colombia. Correo electrónico: meliana.cardona@udea. edu.co. Código Orcid: https://orcid. org/0000-0002-3697-2288

** Magíster en Educación. Profesor, Facultad de Educación, Departamento de Enseñanza de las Ciencias y las Artes, Universidad de Antioquia, Colombia. Correo electrónico: jandres.carmona@udea.edu.co. Código Orcid: https://orcid. org/0000-0002-0100-7090

*** Magíster en Educación en Ciencias Naturales. Profesora, Facultad de Educación, Departamento de Enseñanza de las Ciencias y las Artes, Universidad de Antioquia, Colombia. Correo electrónico: vanessa.arias@ udea.edu.co. Código Orcid: https:// orcid.org/0000-0002-9449-6144

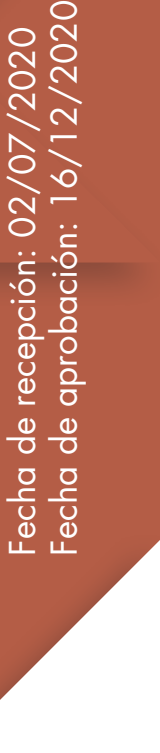




\begin{abstract}
Teaching professional identity is a fundamental axis in teacher training and its study from the first levels of training allows understanding its structure and impact on pedagogical practices. The purpose of this research was to characterize some traits of professional identity that students of a Bachelor program in Physics have. It is based on their perceptions and expectations about the teaching profession; as well as assessing the contribution of the course Pedagogical Practice I in recognizing the components in the construction of identity. The approach was biographical-narrative using letters and self-assessment as instruments for recording student perceptions. The main finding is the transformation of the students' conceptions about teaching profession. They achieved to identify different components in the construction of identity and apply them in their own recognition as pre-service teachers.
\end{abstract}

Keywords:

teacher training; pedagogical practice; practicum; professional identity; teaching profession

\title{
Resumo
}

A identidade profissional docente é um eixo fundamental na formação de professores, seu estudo desde os primeiros níveis de formação permite compreender sua configuração e incidência nas práticas pedagógicas. O objetivo desta pesquisa foi caracterizar os traços de identidade profissional que os estudantes que ingressam no curso de bacharelado em Física possuem, com base em suas percepções e expectativas sobre a profissão docente; assim como avaliar as contribuições do espaço de formação da Prática Pedagógica I no reconhecimento da construção da mencionada identidade. O estudo foi desenvolvido sob uma abordagem biográfico-narrativa e recorreu à elaboração de cartas e a autoavaliação como instrumentos para registrar a informação. A principal constatação é a transformação das concepções dos estudantes sobre a profissão docente, de forma que eles conseguiram reconhecer os diferentes componentes da construção da identidade e aplicá-los em seu reconhecimento como futuros professores.

Palavras-chave:

formação de professores; prática pedagógica; prácticum; identidade profissional; profissão docente 


\section{Introducción}

Los programas de formación de profesores en Colombia han tenido modificaciones curriculares a lo largo de la historia en atención principalmente a las necesidades de la sociedad; es reciente la transformación que tuvo especial impacto en la Licenciatura en Matemáticas y Física, motivada por el Decreto 2450 del 2015 y la Resolución 02041 del 2016 del Ministerio de Educación Nacional. Esta transformación implicó un cambio de denominación de los programas de formación pasando a atender solo una de las áreas específicas, en este caso la Física. Como parte del proceso, se incluyeron en el plan de estudios prácticas pedagógicas tempranas, un cambio de gran valor si se considera que estos espacios estaban ubicados principalmente al final del proceso de formación.

En la Licenciatura en Física de la Universidad de Antioquia, estos espacios se han concebido como una oportunidad para la integración de saberes disciplinares, pedagógicos y didácticos, y algunos de los propósitos formativos que los orientan son: 1) el reconocimiento de la identidad profesional, 2) la epistemología de la enseñanza de la física, 3) los ambientes educativos no convencionales, 4) los recursos tecnológicos y 5) la atención a la diversidad. El interés del presente trabajo se enfocó en el curso Práctica Pedagógica I (en adelante, PP-I), el cual tiene como propósito favorecer la reflexión y el desarrollo del pensamiento crítico sobre la configuración de la identidad profesional, aspectos que ahora se proyectan como una oportunidad para complementar la formación profesional y favorecer la permanencia de los estudiantes en el Programa.

En virtud de lo anterior, se orientó el desarrollo del presente estudio a: 1) caracterizar los rasgos de identidad profesional que tienen los estudiantes que ingresan a un programa de Licenciatura en Física, a partir de sus percepciones y expectativas sobre la profesión docente, y 2) valorar los aportes del espacio de formación PP-I en el reconocimiento de los componentes de la construcción de dicha identidad.

\section{Antecedentes}

En la literatura existen pocos trabajos que indagan acerca de la construcción de la identidad profesional del profesor de física; sin embargo, se reconoce el valor de las investigaciones producidas en relación con la identidad profesional del profesor de ciencias, ya que comparten en esencia la conceptualización sobre la enseñanza y el aprendizaje del conocimiento científico (Cardona et al., 2019). Además, se ha reconocido que la identidad sirve como lente para estudiar el aprendizaje de las ciencias y para la preparación de los profesores, al brindar contextos seguros donde pueden construir sus identidades de enseñanza de ciencias y para apoyar su participación en diversas comunidades de aprendizaje (Avraamidou, 2014; Vanegas y Fuentealba, 2019).

Por su parte, las investigaciones en relación con la práctica pedagógica contemplan algunas preocupaciones sobre la manera de aproximar a los profesores en formación a los procesos de investigación (Zapata y Vélez, 2008), considerando que este escenario es fundamental en el reconocimiento de la observación, reflexión, inmersión e investigación como múltiples factores que se interrelacionan y favorecen nuevas formas de desarrollar la práctica profesional (Cristovão et al., 2018; Fabro, 2016). Estos factores permiten la apropiación de los fundamentos de la identidad profesional docente y la compresión del 
sentido formativo de los contextos propios del desempeño de su profesión (Bolívar, 2006; Sayago et al., 2008).

En este sentido, la práctica pedagógica debe ser una experiencia para quien pretende enseñar (Espinosa, 2013), una experiencia que articule diferentes dimensiones y reconozca la investigación como un elemento fundamental; de esta manera "podrá darse mayor acercamiento al ideal de un maestro que tiene como característica esencial el desarrollo de su hacer profesional vinculado con la transformación de los contextos" (Jaramillo, 2007, p. 12).

\section{Marco teórico}

\section{Identidad profesional de profesores}

La identidad profesional de profesores se construye a través de un conjunto de dinámicas que cobran sentido por medio de la experiencia (Bolívar, 2006). Estas dinámicas dan lugar a la reflexión sobre condiciones políticas, económicas, sociales y culturales que determinan las posturas pedagógicas y didácticas que orientan el sistema educativo en un contexto y época determinada. Por ello, una de las principales estrategias implementadas en la Licenciatura en Física es reconocer, a través de las historias de vida de los profesores en formación y de quienes están en ejercicio, algunos componentes de la identidad profesional. En el cuadro 1 se describen dichos componentes, tomando como referente a Bolívar et al. (2005).

\section{Cuadro 1. Componentes de la identidad profesional}

\begin{tabular}{ll}
\hline $\begin{array}{c}\text { Componente de la } \\
\text { identidad }\end{array}$ & \multicolumn{1}{c}{ Descripción } \\
\hline Autoimagen & $\begin{array}{l}\text { La autoimagen es el "modo en que el sujeto se define a sí mismo y, a la } \\
\text { vez, es definido poseyendo ciertas características, idénticas o específicas } \\
\text { suyas, en relación con otros individuos dentro de la profesión" (Bolívar, } \\
\text { 2006, p. 129). }\end{array}$ \\
\hline Reconocimiento social & $\begin{array}{l}\text { Tiene que ver con la forma como otras personas (padres de familia, } \\
\text { familiares, amigos, estudiantes y otros colegas) ven la profesión. }\end{array}$ \\
\hline Grado de satisfacción & $\begin{array}{l}\text { Se refiere a las motivaciones, competencias, agrado y situaciones que } \\
\text { proporcionan bienestar, vinculadas con la naturaleza de su trabajo. }\end{array}$ \\
\hline Relaciones sociales & $\begin{array}{l}\text { Son las relaciones con colegas, estudiantes o demás integrantes de una } \\
\text { comunidad educativa. }\end{array}$ \\
\hline Actitud ante el cambio & $\begin{array}{l}\text { Representa un reto personal que implica cambios en diferentes } \\
\text { dimensiones y etapas de la formación inicial. }\end{array}$ \\
\hline $\begin{array}{l}\text { Competencias } \\
\text { profesionales }\end{array}$ & $\begin{array}{l}\text { De acuerdo con Bolívar (2007), los conocimientos y competencias de los } \\
\text { docentes son los que conducen a su profesionalización y a la cualificación } \\
\text { de sus prácticas. }\end{array}$ \\
\hline
\end{tabular}


Expectativas a futuro Se refiere a aquello que los maestros desean a futuro y que está se

relaciona de forma directa con las expectativas tanto personales como

profesionales (Bolívar, 2006).

Fuente: elaboración propia. Con base en Bolívar 2006; 2007

En este sentido, la configuración de la identidad profesional es un proceso que involucra componentes cognitivos, sociales y afectivos, "donde los valores personales y profesionales están en el núcleo que, a su vez, se expresa en la motivación, actitud y compromiso con que el profesorado afronta su trabajo" (Bolívar, 2007, p. 14). El reconocimiento de estos componentes se constituye en un factor clave para comprender, entre otras cosas, las percepciones de los futuros profesores para la elección y permanencia en los programas de formación. Dado que el primer año constituye un punto nodal en la decisión de abandonar o proseguir los estudios, se hace necesario conocer a los estudiantes que ingresan a las licenciaturas para diseñar programas de apoyo y estrategias que transformen su compromiso y persistencia (Silva, 2011).

\section{Metodología}

\section{Contexto de la investigación}

El presente estudio se llevó a cabo con dos grupos de estudiantes del curso de PP-I de la Licenciatura en Física de la Universidad de Antioquia. Incluyó un seguimiento longitudinal a partir de estrategias que fueron implementadas y registradas en los dos semestres del 2019. En el primero, los participantes fueron 18 estudiantes (cuatro mujeres y catorce hombres) $y$, en el segundo 13 estudiantes (tres mujeres y diez hombres). Este espacio de formación da comienzo a las Prácticas Pedagógicas tempranas del programa de Licenciatura en Física y contempla algunas estrategias metodológicas que se describen en el cuadro 2.

Cuadro 2. Estrategias metodológicas utilizadas en el curso

\begin{tabular}{ll}
\hline \multicolumn{1}{c}{ Instrumento } & \multicolumn{1}{c}{ Descripción } \\
\hline $\begin{array}{l}\text { Narrativas e historias } \\
\text { de vida }\end{array}$ & $\begin{array}{l}\text { Los estudiantes analizan cómo su práctica cotidiana se permea } \\
\text { por las experiencias previas y las reflexiones generadas a partir de } \\
\text { las discusiones en el curso (Bolívar, 2012). Además, a partir de la } \\
\text { elaboración de dos cartas "al yo del futuro" (al iniciar y al finalizar el } \\
\text { curso), es posible conocer su proyección como docentes a largo plazo. }\end{array}$ \\
\hline Autoevaluación & $\begin{array}{l}\text { Proporciona información sobre la valoración de la práctica pedagógica y } \\
\text { la contribución a la formación de los estudiantes desde el reconocimiento } \\
\text { de los elementos que constituyen la identidad profesional del docente de } \\
\text { ciencias y de física, la valoración histórica del proceso de configuración } \\
\text { identitaria del docente de ciencias en Colombia y las características del } \\
\text { desarrollo profesional como integración a la reflexión sobre la práctica } \\
\text { docente. }\end{array}$ \\
\hline
\end{tabular}




\section{Enfoque}

El presente trabajo se desarrolló bajo un enfoque biográfico-narrativo, considerando que dentro de la investigación cualitativa se contempla la narrativa como contextual y específica, y consiste en recrear las diferentes concepciones que puede evocar una persona sobre su trayectoria de vida, a partir del registro y análisis de relatos que ayudan a comprender el objeto de estudio (Cardona y Chaurra, 2018). De acuerdo con Sayago et al. (2008), contar la propia historia implica un proceso de reflexión y autoconocimiento, que son relevantes en la construcción de la identidad; por lo tanto, se favorecieron las percepciones de la profesión docente y sus expectativas a futuro, para identificar los componentes de la identidad profesional de los docentes en formación inicial.

Para efectos del presente artículo, se consideraron como instrumentos para el registro de la información: dos cartas elaboradas por los estudiantes que estaban dirigidas a su "yo del futuro" (una al inicio y otra al finalizar el curso) y la autoevaluación final (véase cuadro 2). La elaboración de las cartas se propuso al inicio del curso (primera clase) como una actividad individual, de manera privada y con un tiempo de una semana para su elaboración. Luego, se planteó la misma dinámica al finalizar el curso, para realizar la entrega en la última clase. En ninguno de los dos momentos se socializaron las cartas. La autoevaluación tenía el fin de identificar su valoración en relación con el aporte del curso al proceso de formación, las habilidades que se fortalecieron, y la comprensión sobre los ejes temáticos que se abordaron. Teniendo en cuenta el cumplimiento con la elaboración de dichos instrumentos y las características propias de un estudio cualitativo en relación con el número de casos considerados, se seleccionaron 12 participantes (seis de cada grupo, identificados como E1, E2,... hasta E12) para ser analizados en profundidad, sin el propósito de generar extrapolaciones a otros contextos. Asimismo, atendiendo a las consideraciones éticas de la investigación, cada uno de ellos firmó un consentimiento informado para autorizar el uso de datos.

\section{Análisis de la información: la sistematización como una mirada de saberes movilizados en la Práctica Pedagógica I}

La sistematización es un apoyo metodológico sustancial en la producción de conocimiento en la formación de profesores y permite identificar otros lugares y formas de comprender las transformaciones curriculares que afrontan las licenciaturas (Carmona-Mesa et al., 2018). En particular, la sistematización como mirada de saberes visibiliza los procesos y las prácticas presentes en las reflexiones gestadas en la PP-I, como una producción colectiva de nuevas miradas en la formación inicial de profesores (Mejía, 2008).

En el proceso de sistematización, análisis e interpretación de la información se categorizaron y codificaron (Cisterna, 2005) las cartas elaboradas por los futuros 
profesores y la autoevaluación diligenciada al finalizar el curso, lo cual permitió identificar los componentes clave de la construcción de identidad a partir de su proyección a futuro (cuadro 1), que fueron definidos como categorías para el análisis. De esta manera, por medio de la triangulación entre investigadores, y con el marco teórico, de los registros de dichas cartas y su respectiva autoevaluación, se logró caracterizar estos componentes en sus percepciones (Bolívar et al., 2005) y expectativas sobre la profesión docente, así como valorar los aportes del espacio de formación en su reconocimiento.

\section{Resultados y análisis}

A continuación, se describen los principales rasgos identificados a través de los instrumentos y se analizan a la luz de las percepciones y expectativas sobre la profesión docente.

\section{Autoimagen}

Este componente responde a la pregunta sobre cómo se ve el estudiante en la Licenciatura que está cursando. De esta manera, va a estar marcado por la trayectoria que tenga la persona y va de la mano con el reconocimiento social de la profesión. En la mayoría de los participantes se identificó que hacían alusión a la docencia como un asunto vocacional o se referían a la importancia de la relación pedagógica entre profesores y estudiantes:

- $\quad$ "Elegiste tras un proceso de aprender y conocerte que este es tu papel en la vida, el de enseñar conocimientos que tanto te apasionan y enriquecen la vida" (E7, Carta 1). ${ }^{1}$
- " "...] ha recapturado la esencia de ser maestro, les ha mostrado a los estudiantes lo fascinante que suele ser encontrar una respuesta a fenómenos que no imaginábamos que existieran; y a su vez, ha sembrado la curiosidad en aquellas mentes ávidas que intentan dominar el mundo" (E3, Carta 1).

- "Tienes la mayor satisfacción al saber que sigues siendo tú y de paso aplicaste a tu vida cualidades de las personas que te indujeron a escoger esta profesión que llena tu vida de vida" (E9, Carta 1).

En las expresiones anteriores, se observan concepciones iniciales favorables sobre el papel que quieren ejercer como futuros profesores, en las que se puede reconocer algunos rasgos de su identidad que han sido producto de lo que esperan de un profesor. Por otra parte, se identificaron algunos fragmentos en la carta inicial de dos participantes (E1, E5), que dan cuenta de que ingresaron al Programa sin que fuera algo que los motivara o lo hicieron con desconocimiento de la profesión; sin embargo, se muestra sensibilidad y disposición para considerar su ejercicio profesional. Al comparar con las cartas finales de ambos, se observa una transformación en la percepción de la profesión (véase cuadro 3), que favorece la consolidación de otros rasgos identitarios, como las expectativas a futuro y las competencias profesionales. 


\begin{tabular}{|c|c|c|}
\hline Estudiante & Carta inicial (carta 1) & Carta final (carta 2) \\
\hline El & $\begin{array}{l}\text { "Sé que no fue tu primera } \\
\text { opción, sé que ser maestro } \\
\text { no era tu vocación principal, } \\
\text { pero si por medio de la } \\
\text { ciencia quieres cambiar el } \\
\text { mundo, por medio de la } \\
\text { docencia también lo puedes } \\
\text { hacer". }\end{array}$ & $\begin{array}{l}\text { "Me di cuenta de que la labor del maestro no } \\
\text { es más que la labor más ardua de este mundo } \\
\text { entero. Este primer semestre me enseñó, como } \\
\text { nunca, a valorar al profesor, porque me enfrenté } \\
\text { con muchas realidades que nunca antes había } \\
\text { concebido sobre ejercer la docencia [...] eres } \\
\text { un ser humano íntegro, culto y sensible, que } \\
\text { por medio de la ciencia está al servicio de la } \\
\text { sociedad, lo cual me hace pensar que todo va } \\
\text { por buen camino". }\end{array}$ \\
\hline E5 & $\begin{array}{l}\text { "¿Aún recuerdas la primera } \\
\text { clase de práctica? El cómo } \\
\text { reflexionaste sobre la } \\
\text { imagen tuya como maestro } \\
\text { y te cuestionabas si era tu } \\
\text { vocación". }\end{array}$ & $\begin{array}{l}\text { "Lo más impactante del viaje que he emprendido } \\
\text { a lo largo de estos meses fue comprender la } \\
\text { complejidad del trabajo docente. Antes del curso } \\
\text { jamás se me hubiese podido pasar por la cabeza } \\
\text { que enseñar puede ser algo tan complejo, tan lleno } \\
\text { de matices y de puntos de vista distintos. Jamás } \\
\text { pensé que me iría con tantas preguntas. ¿̇Cuál } \\
\text { es el papel del estudiante en todo su proceso de } \\
\text { aprendizaje? ¿̇El mío como docente? ¿Quién soy yo } \\
\text { como docente y qué puedo ofrecer?" }\end{array}$ \\
\hline
\end{tabular}

Fuente: elaboración propia con base en las cartas de los participantes.

Los argumentos consignados en los registros de ambos estudiantes permiten intuir que el curso favoreció la valoración de la profesión y su autoimagen; esto puede relacionarse además con la respuesta que en la autoevaluación dio E5 a la pregunta żcuál fue el aporte del curso a su proceso de formación? Según el estudiante, "básicamente el curso ha representado las bases de la pregunta más importante que me propuse responder durante la carrera, ¿Quién soy yo como docente?". En esta respuesta se identifica que el aporte fue más allá de lo conceptual y trascendió al conocimiento personal.

Por otra parte, en los siguientes fragmentos de E3 y E 11 se identifica un carácter reflexivo de su proyección como seres humanos íntegros, intelectuales y formados profesionalmente, en una práctica docente que tenga sentido y coherencia con su proyecto identitario y el de los demás. Además, que les permita desenvolverse en un espacio en constante transformación, en el que "se construyen comunidades, se comprende y elaboran relaciones con los demás y con el mundo, y además, se adquiere el sentido vivido de lo que se es" (Vanegas y Fuentealba, 2019, p. 121).

- "A pesar de todo lo bueno, usted conocerá aquellos aspectos que debe mejorar; esos límites y frustraciones que lo han frustrado en algún momento, pero que con el tiempo ha sabido valorar para mejorar su vocación; le invito a que no desfallezca y entienda que su profesión es el pilar de la sociedad" (E3, Carta 2). 
- "Espero que seas altamente congruente entre tus palabras y tus acciones, tomamos la decisión consciente de no solo conocernos por dentro, sino de expresar todo lo que somos con nuestros actos. Mírate, piénsate, analízate, reflexiónate, actúa, cambia, mejora siempre y nunca pares de hacerlo" (E 1 1, Carta 2).

Por otra parte, cabe resaltar el aporte de uno de los participantes, quien al finalizar el curso llegó a un punto de convergencia entre los elementos mencionados anteriormente (vocación, relación pedagógica, rol en la sociedad):

- "De lo que sí tengo total certeza es que este es mi camino, que me identifico con lo que representa ser un docente, con su rol en la sociedad, con sus áreas de trabajo, quizás en un principio esto que digo no era tan claro, pero cómo lo iba a ser si no había tenido ese primer acercamiento con lo que es ser un docente, con la identidad profesional como un proyecto, con la docencia" (E12, Carta 2).

Así pues, es importante resaltar que este grupo de participantes se ven a ellos mismos como docentes y que en estos pensamientos han influido tanto visiones internas como ex- ternas de la profesión; como lo afirma E2 en la autoevaluación: "este curso me aportó mucho reconocimiento propio, dándome confianza expresiva y mucho más control a la hora de compartir con las demás personas y también un entendimiento claro de la carrera escogida" (E2). Esto evidencia que en su primer semestre algunos no estaban seguros de querer ser docentes, pero tuvieron una transformación en sus concepciones iniciales sobre la profesión que les otorgó mayor claridad y fundamentación sobre lo que representa; de acuerdo con Vanegas y Fuentealba (2019), esto ayuda a fortalecer el enfoque reflexivo en la formación de los profesores.

\section{Reconocimiento social}

El reconocimiento social es quizás uno de los componentes más importantes en el proceso de la constitución de la identidad docente y se va constituyendo a partir de las identidades de los demás, lo cual permite un cruce de miradas de la profesión tanto externas como internas (Bolívar, 2006). En este ejercicio investigativo, se identificaron algunas percepciones de los participantes en cuanto al reconocimiento social de la profesión desde diferentes dimensiones. Para la mayoría, este rasgo estuvo presente en la segunda carta y en la autoevaluación (véase cuadro 4).

Cuadro 4. Expresiones de los participantes relativas al reconocimiento social

\begin{tabular}{|c|c|}
\hline Expresiones de los participantes & Interpretación \\
\hline $\begin{array}{l}\text { - "Me enteré, en este primer nivel, que con la formación de } \\
\text { maestros íntegros y de calidad y la defensa de la educación como } \\
\text { instrumento de cambio social, se puede alcanzar un nivel alto en } \\
\text { enseñanza e investigación, que nos llevé a una mejor Colombia" } \\
\text { (E1, Carta 2). } \\
\text { - "La realidad de ser un docente de física ha logrado tener unos } \\
\text { caminos fuertes logrando así un reconocimiento propio del saber } \\
\text { que se tiene, de la pasión que se tiene inculcada y del deseo de } \\
\text { poder cumplirla" (E2, Carta 2). }\end{array}$ & $\begin{array}{l}\text { Reconocimiento que ha } \\
\text { logrado tener la docencia } \\
\text { en física históricamente } \\
\text { en Colombia, y cómo se } \\
\text { le otorga importancia al } \\
\text { aprendizaje de esta ciencia } \\
\text { en la sociedad. }\end{array}$ \\
\hline
\end{tabular}

\section{en la autoevaluación (véase cuadro 4).}


- "El curso permitió una primera inmersión a lo que es la enseñanza de la física en Colombia. Así pues, me siento preparado para hablar y leer de enseñanza de la física, al menos, en mi contexto colombiano" (El, Autoevaluación).

- "Cabe destacar la manera a la que te refieres de la profesión, siempre hay palabras positivas cuando hablas de ella, te identificas de una manera impresionante con la carrera de licenciatura, la matemática y la física te han permitido crecer como persona, te han alimentado de valores, conocimientos, experiencias, etc." (E6, Carta 2).

- "Aprendí demasiado sobre lo que implica esta formación inicial y de los futuros cambios que puedo tener en lo personal, aprendí que la pasión es algo que se contagia" (E12, Carta 2).

- "Afianza conocimientos previos y aporta nuevos al ejercicio de la profesión docente, contextualiza y ayuda al reconocimiento del sujeto dentro de la identidad docente" (E3, Autoevaluación).

- "No olvides el valor de la enseñanza y la deuda que tienes con aquellas personas que te formaron de una manera amable con sus conocimientos, sus saberes. Quiero que tú, yo futuro, no te olvides de tu origen, y para eso te escribo, recuerda todo lo que llevas detrás" (E4, Carta 2).

- "Usted ha logrado crear el nivel de confianza necesario para que entiendan que su identidad como docente, su reconocimiento de roles y su separación de vida profesional y laboral han sido exitosos, y lo han llevado a lograr aquellos triunfos que hoy como docente le preceden [...] quiero felicitarle porque reconoce contextos identificando las necesidades particulares - a grandes rasgos - de todos aquellos sujetos en formación que usted tiene a su cargo; ha creado en ellos lazos de liderazgo y respeto, de amor y disciplina, fundamentales para la realización profesional y la práctica pedagógica" (E3, Carta 2).

Fuente: elaboración propia con base en las cartas de los participantes.

En el cuadro 4 se describen diferentes factores que influyen en la constitución de este rasgo identitario; desde el rol del profesor en la sociedad, la importancia de su formación inicial y continuada, y el papel de otras personas que valoran como parte de su formación o que están vinculadas de forma directa con su futura labor. Estos comentarios están en coherencia con lo que plantea Bolívar (2006), para quien el bajo reconocimiento que tienen en la actualidad los docentes va de la mano con un sentido de poca valorización de la profesión, lo que repercute también en la autoimagen de algunos de ellos. Por otra parte, se resalta que algunos participantes (E1, E3) valoraron significativamente el aporte del curso en relación con el reconocimiento de la configuración histórica de la enseñanza de la física en Colombia y el papel de la ciencia en la identidad docente; estos ejes fueron desarrollados durante las reflexiones que tuvieron lugar en el espacio de formación.

\section{Competencias profesionales}

Bolívar (2007) argumenta que los conocimientos y las competencias de los docentes conducen a su profesionalización y a la cualificación de sus prácticas. Por esta razón, este componente se relaciona ampliamente con los planteamientos
Reconocimiento de la como futuros profesores. sobre lo que significaba un programa de licenciatura mejor apreciación de la y el fortalecimiento de su vocación.

Reconocimiento de la profesión para la familia y para otros educativa, como los padres de familia y los colegas. 
de algunos autores como Shulman (1987), y Amórtegui y Mosquera (2018), entre otros, quienes se han interesado por mostrar las dimensiones desde las cuales un profesor construye su conocimiento. Como lo menciona Perafán, "el profesor no sólo construye un saber profesional sobre el cómo enseñar, sino también un saber disciplinar alrededor del qué enseñar" (2013, p. 2). Esto da una idea sobre lo que se debe fortalecer en la formación en cuanto a identificar qué y cómo un profesor construye su conocimiento.

En relación con lo anterior, se contemplaron algunas concepciones de Shulman (1987) sobre el conocimiento profesional del profesor, quien señala los siguientes componentes: "el conocimiento del contenido; el conocimiento didáctico general; el conocimiento del currículum; el conocimiento didáctico del contenido; el conocimiento sobre los alumnos, y el conocimiento de los objetivos, las finalidades y los valores educativos" (Castañeda y Perafán, 2015, p. 14). En las expresiones de los participantes que se referían a las competencias profesionales fue posible identificar una aproximación a las características de dichos conocimientos (véase cuadro 5).

Cuadro 5. Componentes del conocimiento del profesor identificadas en el registro de la información

- "La verdad quiero aspirar a ser un profesor lleno de conocimiento total el cual pueda enseñar más de lo que te exigen, poder responder cualquier inquietud o conocimiento extra que tal vez un estudiante quiera adquirir por su interés frente a la materia" (E2, Carta 1).

- "Continúo exaltando su conocimiento en su ciencia, allí demuestra la gran experiencia que tiene y el amor que le pone a lo que hace" (E6, Carta 1).

- "Recuerda cuidar tu relación con el saber, pues es esto lo que enseñarás; fortalécela y no dejes que se estanque. Piensa tu ciencia y tu profesión para construirlas" (E7, Carta 2)
Conocimiento del contenido

- "Durante las últimas semanas he notado que su pedagogía experimental ha desarrollado en los estudiantes aquella pasión que creíamos perdida por las ciencias naturales, especialmente por un Conocimiento didáctico área que normalmente es considerada "aburrida" como lo es la física" (E3, Carta 1).

- "Ha sido una apertura interesante al proceso de construcción de mi identidad como docente a través del estudio de la relación entre docencia y ciencia a través de la historia en nuestro país, así como las diferentes concepciones de ciencia y de la labor educativa" (E7, Autoevaluación).

- "Y tú como maestro despierta esa curiosidad, ese interés en tus estudiantes, haz que sean creativos e innovadores, que se formen con un pensamiento crítico" (E4, Carta 2).

Conocimiento de los alumnos

- "Debes tener siempre en mente que tú también estás en el aula para aprender. Estás ahí, de pie, dándole la espalda a un tablero para aprender a ser lo que todo maestro necesita: ser humano" (E5, carta 1).

- "Es gracias a este curso que entendí que el estudio y la investigación van de la mano con la docencia, y es por eso que un profesor es antes estudiante" (E8, Carta 2).

- "Si alguien me preguntase qué se necesita para ser maestro en este momento, seguramente responderé algo como 'Un poco de todo. Los maestros tienen ganado el cielo' pues no hay profesión más noble que la de la enseñanza" (E5, Carta 2).

- "Creo que este curso es importante para mi formación ya que me dejó ver el verdadero sentido de ser una docente de física, de su historia, de las luchas docentes, de cómo hacer investigación pedagógica y cómo se ha hecho a lo largo de la historia" (E3, Autoevaluación).
Conocimiento de los objetivos, las finalidades y los valores educativos 
En las expresiones del cuadro 5 se evidencian algunos componentes del conocimiento del profesor que hacen énfasis en el saber disciplinar (conocimiento del contenido) y en el reconocimiento de los objetivos de la práctica docente, lo que correspondería a la imagen que tienen inicialmente de la formación académica en la disciplina a enseñar y de la estructura de la profesión docente. No obstante, cabe resaltar que no se identificó el conocimiento sobre el currículo "el cual alude a la familiaridad con las formas de organizar y de dividir el conocimiento" (Perafán, 2013, p. 7), dado que aún no se han familiarizado con el ejercicio de la profesión. Así mismo, en las expresiones de algunos participantes (E3, E7) se da cuenta de una valoración positiva en relación con la apropiación que lograron sobre conceptos como identidad, profesión docente, investigación y ciencia.

En general, los fragmentos relacionados con este componente ayudan a valorar el proceso de formación como un espacio para reconocer los principales elementos de la identidad profesional de un profesor de ciencias, y de física en particular, a partir de la articulación entre su dimensión personal, el acercamiento al conocimiento científico, al conocimiento didáctico y el desarrollo profesional docente. De igual manera, se resalta que el logro de este propósito estuvo acompañado del fortalecimiento de habilidades que los participantes consideran fundamentales para su formación. Principalmente, en la autoevaluación destacaron que, gracias a este espacio, desarrollaron habilidades de lectura crítica, argumentación (E1, E6, E7, E1 1, E12); escucha (E2, E7, E8); trabajo en equipo (E3, E10, E12); pensamiento crítico (E5, E7, E8, E12); oralidad (E7, E10); escritura reflexiva $(E 7, E 9, E 11)$; reconocimiento de estrategias de enseñanza $(E 3, E 8)$.

\section{Expectativas a futuro}

De acuerdo con Bolívar (2006), las expectativas tienen que ver con aquello que se desea a futuro, tanto a nivel personal como profesional; en este sentido, la totalidad de los participantes hizo alguna mención respecto a este componente. A partir de la elaboración de las cartas fue posible identificar cómo se proyectan los futuros profesores en el campo profesional, los retos a los que creen que se enfrentarán y los logros que esperan cumplir. En el cuadro 6 se describen estas características. 
- "Reconozco que todo esto ha sido un viaje de autoconocimiento, un enfrentamiento contra mí mismo y contra las cuestiones más primordiales como mi identidad. Me siento ansioso por ver en qué clase de profesor puedo convertirme algún día en el futuro" (E5, Carta 2).

- "Aunque inicialmente no estaba seguro de si quería ser docente de Física o no, con el tiempo entendí que la base sobre la cual me formara era lo de menos, que lo que me apasiona va más allá de ese contenido que le pueda dar a mis futuros alumnos, que lo que me gusta y me encanta de esto es el impacto que puede generar en una persona un docente" (E12, Carta 2).
En estas expresiones se identifica que los participantes no se veían al inicio como profesores, por motivos como: inclinación por el saber disciplinar, desconocimiento de la profesión, reconocimiento social. Pero al finalizar el curso, se logró una transformación en sus concepciones sobre la práctica docente que, si bien no deja de generar incertidumbre, da cuenta de un fortalecimiento en su vocación.
- "En un futuro planeo o me veo como un maestro lleno de capacidades y saberes indudables, pero principalmente como uno que sabe defenderse o adaptarse a cualquier tipo de ambiente, siendo así un docente con las capacidades de adaptación en el contexto que está trabajando" (E2, Carta 2).

- "Quiero resaltar esa labor como pedagogo en el avance investigativo en su campo disciplinar, lo cual beneficia de manera directa a los sujetos en formación, a la institución y al rescate del renombre de docente como figura investigativa basada en modelos desarrollistas y generando ese interés en la comunidad para superar sus límites y crear un cambio en el contexto en el que cada uno se desenvuelve" (E3, Carta 2).

- "Espero que seas un gran maestro, como aquellos que tuviste y que de aquellos que no quisiste hayas aprendido, lo que no debes repetir. Que seas un maestro del que te sientas orgulloso y del que te gustaría aprender" (E7, Carta 1).

- "Espero entonces, que estemos realizando ese humilde sueño de estar en un lugar alejado de la ciudad y sus ataduras, viviendo al lado de la escuelita en la cual enseñas, ayudando a niños y jóvenes a descubrir el mundo un poco más, a interesarse por la ciencia: física, astronomía, biología; o por los números o por cualquier cosa que ellos quieran aprender" (E1 1, Carta 1).
Estos participantes indican que a futuro se ven como profesores ejemplares, profesionales íntegros, que fomentan el pensamiento crítico, identifican las necesidades en sus contextos y establecen vínculos con sus estudiantes a través del amor por el conocimiento.

Fuente: elaboración propia con base en los escritos de los participantes.

En los fragmentos del cuadro 6 se evidencia que el espacio de PP-I logra impulsar en los estudiantes no solo su autoimagen sino sus expectativas a futuro; este es un asunto fundamental, ya que se encuentran en primer semestre y este es quizá su primer acercamiento a la docencia. Se resalta la importancia que le otorgan a la investigación y a la formación posgraduada (E3, E6) para la cualificación de la práctica docente y el reconocimiento social de su labor, aspectos coherentes con los propósitos de formación del Programa.
En estos fragmentos se refieren situaciones particulares que influyen directamente en sus expectativas a futuro, como lo son el referente de profesores que marcaron su trayectoria y el sueño de impactar contextos rurales. 
Cuadro 7. Valoración del curso en el reconocimiento de los componentes de la identidad profesional

\begin{tabular}{|c|c|}
\hline Expresiones de los participantes & Interpretación \\
\hline $\begin{array}{l}\text { - "Constantemente estuvimos reconociéndonos a } \\
\text { nosotros mismos como futuros profesores de física, y } \\
\text { considero que de ahí parten todos los aspectos que } \\
\text { conforman la identidad profesional" (E9). } \\
\text { "Considero que uno de los sentidos más importantes } \\
\text { de este curso es precisamente encontrar la verdadera } \\
\text { identidad profesional docente, sin dejar de lado que } \\
\text { siempre será un trabajo individual y personal" (E1 1). }\end{array}$ & $\begin{array}{l}\text { En estas expresiones se resalta que la } \\
\text { metodología de las clases a partir de las } \\
\text { discusiones y talleres contribuyó a que } \\
\text { aprendieran a cuestionarse y a valorar la } \\
\text { construcción colectiva de conocimiento. }\end{array}$ \\
\hline $\begin{array}{l}\text { - "Me di cuenta de que es importante pensarnos y } \\
\text { pensar a los demás para que así, podamos ir mejorando } \\
\text { nuestra labor docente y nuestro crecimiento personal" } \\
\text { (E1). } \\
\text { - "Este curso es muy pertinente en el primer semestre, } \\
\text { gracias a que constantemente estamos reflexionando } \\
\text { sobre la profesión" (E6). } \\
\text { - "Reflexionar sobre la práctica docente hace que } \\
\text { desarrollemos características o modos en los que } \\
\text { queremos ejercer nuestra profesión, es decir, que nos } \\
\text { permite crear una identidad acerca de qué tipo de } \\
\text { profesores queremos ser" (E9). }\end{array}$ & $\begin{array}{l}\text { Estas respuestas se caracterizaron por } \\
\text { hacer mayor énfasis en la importancia de } \\
\text { la reflexión sobre la práctica docente, lo } \\
\text { que de acuerdo con Vanegas y Fuentealba } \\
\text { "permite aprender de las experiencias } \\
\text { propias y de otros, mejorar la actividad } \\
\text { profesional, producir conciencia sobre las } \\
\text { formas en que se interpreta la realidad y dar } \\
\text { sentido a las acciones y el comportamiento" } \\
(2019, \text { p. 119). }\end{array}$ \\
\hline
\end{tabular}

Fuente: elaboración propia.

A partir de las expresiones descritas en el cuadro 7, se puede afirmar que los participantes valoraron significativamente la metodología del curso porque les permitió un mayor acercamiento a los componentes de la identidad profesional y un proceso autorreflexivo en cuanto a las situaciones que han influido en su decisión de ser profesores. Es posible identificar, además, que los estudiantes consideraron que sus reflexiones permiten pensar en la transformación de sus prácticas y establecer relaciones entre lo que se ha desarrollado históricamente y lo que esperan ser en su futura labor. Lo anterior está en coherencia con lo reportado como un desafío a superar en la formación inicial de profesores (Vanegas y Fuentealba, 2019). En lo relativo a las características del desarrollo profesional y a la reflexión sobre la práctica docente, si bien es una reflexión prematura para el primer nivel del programa, se logró un reconocimiento importante de las diferentes dimensiones del conocimiento del profesor.

\section{Discusión y conclusiones}

En relación con el primer objetivo de esta investigación, el análisis de cada una de las cartas de los participantes y su autoevaluación permitió comprender que tanto lo personal como lo profesional cobran sentido al construir una visión de la profesión cuando apenas se está en formación inicial (Sayago et al., 2008). Al respecto, la metodología utilizada en este trabajo permitió que los participantes expresaran de forma libre sus perspectivas y expectativas frente a la docencia; 
de hecho, la elaboración de las cartas alcanza una relevancia particular como estrategia para la reflexión sobre la práctica pedagógica en la formación de profesores.

Si bien la constitución de la identidad es un proceso que se da a lo largo de la vida, en especial, durante la vida laboral, en la formación inicial se pueden identificar rasgos de estos componentes desde las percepciones de los futuros profesores (autoimagen, reconocimiento social, competencias profesionales, expectativas a futuro); sin embargo, estos se potencian fuertemente en niveles superiores de la formación inicial y en la formación continua. Un ejemplo de esto es que no se identificaron expresiones de los participantes que manifiesten su percepción sobre las relaciones sociales, la actitud ante el cambio y el grado de satisfacción, lo que podría corresponder a la falta de experiencia en el campo laboral y a que gran parte de sus imaginarios se vinculan a situaciones que han vivido en otros contextos desde la observación de otros docentes.

Por otra parte, las percepciones manifestadas por los futuros docentes ayudaron a identificar las principales motivaciones que tienen para permanecer o desertar del programa de formación. Se ha reconocido que el interés por la docencia desempeña un papel importante en el desarrollo de la identidad profesional. Este interés se debería principalmente a motivos de tipo humanista, relacionados con la vocación intrínseca de aquellos que se desempeñan en la educación, o a motivos más pragmáticos, que corresponden a los estereotipos materiales de la profesión como el salario o las vacaciones (Ariza et al., 2011 ). En este sentido, se puede afirmar que la percepción de los futuros docentes de física es algo confusa para algunos, situación que podría relacionarse con el hecho de que se encuentran en el nivel inicial del programa de formación.
Los resultados en relación con la valoración del espacio de formación (objetivo 2) confirman la importancia de las prácticas pedagógicas denominadas como tempranas, pues a partir de la conceptualización en estos espacios se puede atender a posibles motivos de deserción en los programas (Silva, 2011), al indagar por la constitución de su identidad profesional y problematizar el imaginario que se tiene de la profesión docente. Además, se considera pertinente profundizar en estudios que analicen los encuentros o desencuentros en la identidad profesional del profesor de física y de ciencias, que permitan generar espacios de discusión sobre la configuración de la identidad y el desarrollo profesional de los profesores en formación en estas áreas, principalmente como un eje transversal en los cursos del núcleo de Práctica Pedagógica.

Finalmente, se resaltan algunas de las limitaciones del estudio. Por un lado, el tamaño de los casos analizados no permite establecer extrapolaciones con otros contextos, lo que invita en futuras investigaciones a ampliar el alcance; por otro lado, y si bien se reporta una diferencia en relación al sexo de los participantes, este aspecto se reconoce como una limitación en la presente investigación al distar del principal objeto de estudio; por lo tanto, se constituye en una oportunidad para analizar en futuras investigaciones la relación entre sexo e identidad profesional de profesores en programas afines a las ciencias naturales.

\section{Agradecimientos}

Se agradece al Centro de Investigaciones Educativas y Pedagógicas de la Facultad de Educación de la Universidad de Antioquia, por el financiamiento del proyecto "Factores que influyen en la deserción y permanencia 
estudiantil en un programa de Licenciatura en Física" en la Convocatoria interna de investigaciones 2020-2021.

\section{Referencias}

Ariza, L., Sánchez, F. y Pontes, A. (201 1). Motivos de interés por la docencia e identidad profesional: una aproximación a partir de la opinión del futuro profesorado de humanidades y ciencias sociales. Profesorado: Revista de Curriculum y Formación del Profesorado, 15(1), 355-374.

Amórtegui, E. F. y Mosquera, J. A. (2018). Aportaciones de la práctica pedagógica en la construcción del conocimiento del profesor. Tecné, Episteme y Didaxis: TED, 43, 47-65. https://doi.org/10.17227/ted.num43-8651

Avraamidou, L. (2014). Studying science teacher identity: Current insights and future research directions. Studies in Science Education, 50(2), 145-179. https://doi. org/10.1080/03057267.2014.937171

Bolívar, A. (2006). La identidad profesional del profesorado de secundaria: crisis y reconstrucción. Aljibe.

Bolívar, A. (2007). La formación inicial del profesorado de secundaria y su identidad profesional. Estudios sobre Educación, 12, 13-30.

Bolívar, A. (2012). Metodología de la investigación biográfico-narrativa: recogida y análisis de datos. Dimensões epistemológicas e metodológicas da pesquisa (auto) biográfica. EDIPUCRS, 79-109.

Bolívar, A., Fernández, M. y Molina, E. (2005). Investigar la identidad profesional del profesorado: Una triangulación secuencial. Forum Qualitative Social Research, $6(1), 1-27$.

Cardona, M., Villegas, A., Carmona-Mesa, J. y Mejía, L. (2019). Percepción de los maestros en formación de ciencias naturales y física frente a la profesión docente. Revista de Enseñanza de la Física, 31, 159-166. https://revistas.unc. edu.ar/index.php/revistaEF/article/view/2654 1

Cardona, A. y Chaurra, R. (2018). La narración autobiográfica del oficio del maestro: a propósito del libro álbum como posibilidad para repensar la didáctica universitaria. Uni-pluriversidad, 18(1), 47-55. https://doi.org/10.17533/udea. unipluri. 18.1.05

Carmona-Mesa, J. A., Salazar, J. V. y Villa-Ochoa, J. A. (2018). Uso de calculadoras simples y videojuegos en un curso de formación de profesores. Uni-pluriversidad, 18(1), 13-24. https://doi.org/1017533/udea.unipluri.18.1.02

Castañeda, L. A. y Perafán, G. A. (2015). El conocimiento profesional del profesor: tendencias investigativas y campo de acción en la formación de profesores. 
(pensamiento), (palabra)... Y obra, (14), 10 21. https://doi.org/10.17227/2011804X. 14PPO8.21

Cisterna, F. (2005). Categorización y triangulación como procesos de validación del conocimiento en investigación cualitativa. Theoria, 14(1), 61-71.

Cristovão, N. L. L., Alencar, E. S. y Barros, R. A. (2018). Práticas formativas de professores dos anos iniciais do ensino fundamental no grupo práticas pedagógicas em rede (PPR). Uni-pluriversidad, 18(2), 102-119. https:// doi.org/10.17533/udea.unipluri.18.2.07

Espinosa, D. (2013). A propósito de la reflexión de las prácticas pedagógicas y la calidad de la educación. Uni-pluriversidad, 13(2), 119-122.

Fabro, A. P. (2016). Análisis de las prácticas de enseñanza de ciencias naturales de alumnos residentes del profesorado de educación primaria de la Provincia de Santa Fe, Argentina. Uni-pluriversidad, 16(1), 64-75. https:// revistas.udea.edu.co/index.php/unip/article/ view/326185

Jaramillo, R. (2007). La formación investigativa de los maestros: un asunto estratégico para la alta calidad. Uni-pluriversidad, 7(1), 1-13.

Mejía, M. (2008). La sistematización. Empodera y produce saber y conocimiento. Ediciones Desde Abajo.

Ministerio de Educación Nacional. (2016). Resolución 02041.

Perafán, G. A. (2013). El conocimiento profesional docente: caracterización, aspectos metodológicos y desarrollo. En Estado de la Enseñanza de las Ciencias: 2000-2011. MEN-Universidad del Valle.

Sayago, Z. B., Chacón Corzo, M. A. y Rojas de Rojas, M. (2008). Construcción de la identidad profesional docente en estudiantes universitarios. Educere, 12(42), 551-561.

Shulman, L. (1987). Knowledge and teaching: Foundations of the new reform. Harvard Educational Review, 57(1), 1-22.

Silva, M. (2011). El primer año universitario. Un tramo crítico para el éxito académico. Perfiles Educativos, 33(especial), 102-114.

Vanegas, C. y Fuentealba, A. (2019). Identidad profesional docente, reflexión y práctica pedagógica: Consideraciones claves para la formación de profesores. Perspectiva Educacional, 58(1), 1 15-138. http://dx.doi.org/10.4151/07189729-vol.58-iss. 1-art.780

Zapata Vasco, J. J. y Vélez García, L. M. (2008). La investigación en la formación de maestros y maestras. Qué y cómo se enseña y se aprende. Uni-pluriversidad, 8(1), 1-13.

\section{Forma de citar este artículo:}

Cardona, M., Carmona-Mesa, J. y Arias, V. (2022). Percepciones y expectativas profesionales en estudiantes de Licenciatura en Física. Tecné, Episteme y Didaxis: TED, (51). https://doi.

org/10.17227/ted.num51-11988 
